\title{
Assessment of Common Scab-Inducing Pathogen Effects on Potato Underground Organs Via Computed Tomography Scanning
}

\author{
L. Han, P. Dutilleul, S. O. Prasher, C. Beaulieu, and D. L. Smith
}

First, second, and fifth authors: Department of Plant Science, McGill University, Macdonald Campus, 21,111 Lakeshore Road, Ste-Anne-deBellevue, QC H9X 3V9, Canada; first and third authors: Department of Bioresource Engineering, McGill University, Macdonald Campus, 21,111 Lakeshore Road, Ste-Anne-de-Bellevue, QC H9X 3V9, Canada; and fourth author: Département de biologie, Université de Sherbrooke, 2500 Boulevard de l'Université, Sherbrooke, QC J1K 2R1, Canada.

Accepted for publication 11 June 2008.

\begin{abstract}
Han, L., Dutilleul, P., Prasher, S. O., Beaulieu, C., and Smith, D. L. 2008. Assessment of common scab-inducing pathogen effects on potato underground organs via computed tomography scanning. Phytopathology 98:1118-1125.

Common scab caused by Streptomyces scabies is a major bacterial disease of potato (Solanum tuberosum). Its best known symptom is superficial lesions on the surface of progeny potato tubers, observed at harvesting. In this study, effects of $S$. scabies on space occupancy by underground organs and on structural complexity of root systems are investigated

applications, their underground organs and surrounding medium (sieved and autoclaved homogeneous sand) were submitted to CT scanning 4, 6, and 8 weeks after planting. For one group, sand was inoculated with the common scab-inducing pathogen (S. scabies EF-35) at potting. Space occupancy by underground organs was estimated via curve fitting applied to histograms of CT scan data, while three-dimensional skeletal images were used for fractal analysis. Root systems of diseased plants were found to be less complex than those of healthy plants 4 weeks after planting, and the relative growth rates derived from space occupancy measures were of different sign between the two groups from week 4 to week 8 .
\end{abstract} during growth via computed tomography (CT) scanning. Two groups of potato plants were grown in a greenhouse in middle-sized plastic pots. Using a high-resolution X-ray CT scanner formerly used for medical
Additional keywords: fractal dimension, seed potato.
Common scab caused by Streptomyces scabies (Thaxter) Lambert \& Loria is a major bacterial disease of potato (Solanum tuberosum L.) in Canada (22). It is widely distributed across most of the potato growing areas of Africa, Asia, Europe, and South and North America. The best known symptom of the disease is superficial lesions on the surface of potato tubers, which are usually observed at harvesting (14). Common scab control methods include chemical treatment of seed potatoes, irrigation, and soil amendments $(1,27)$. Previous studies have investigated the disease effects by visually estimating the severity of common scab lesions on the progeny tubers at harvesting, that is, after the fact.

As indicated above, common scab-inducing pathogens are known for forming superficial lesions on progeny potato tubers. However, the effect of these pathogens on the seed potatoes and the roots emerging from them has not been extensively studied. One could expect that as a consequence of the infection, the normal uptake of nutrients and water by the plant would be impeded, and that the root system of a diseased potato plant would experience altered growth, compared to that of a healthy potato plant grown in similar conditions of watering, fertilization, and soil medium.

The functioning of the root system at successive developmental stages determines the health status of a plant. A full understanding

Corresponding author: P. Dutilleul; E-mail address: pierre.dutilleul@mcgill.ca

*The $e$-Xtra logo stands for "electronic extra" and indicates that Figure 1 appears in color online.

doi:10.1094/PHYTO-98-10-1118

(c) 2008 The American Phytopathological Society of plant development requires knowledge about root system growth, especially when the soil has uneven resource distribution and restricts nutrient uptake through inadequate nutrient levels. Accordingly, root systems have been at the core of plant studies for more than a century (7).

In addition to functional aspects, architectural characteristics of root systems have long been scrutinized (15). The spatial structure of a root system reflects the development of the plant from the seed and the adaptation of the seedling to the soil medium and community (20,21). Annual crops normally have roots of several orders, with sizes ranging from a fraction of a millimeter to several centimeters in diameter. As a complement to conventional parameters such as root diameter and length, the qualitative concept of complexity has been used to characterize root architecture $(4,8,9,30,31)$

Self-similarity at different scales has been found in many natural structures, including root systems. In fractal analysis, the fractal dimension (FD) is used to quantify the roughness of shapes and the complexity of objects, and to describe the degree to which an object fills space when it meets the fractal assumption of self-similarity. Fractals can be applied to fragmented shapes and objects other than straight lines and planes, resulting in noninteger dimensions. Thus, the FD can be seen as an extension of the dimensions 1, 2, and 3 of classical Euclidean geometry.

Root systems are good candidates for fractal analysis because of their branching process during growth. The FD takes both the roughness of individual roots and the total number of branches into account $(5,8)$. Thus, the FD of a root system not only characterizes the stage of development of the plant (e.g., juvenile versus adult), but also its responses to water and nutrient availability and environmental stress and heterogeneity $(6,15,33)$. 
Prior to the advent of three-dimensional imaging techniques, fractal analysis in root system studies had to be performed from two-dimensional projections made in given directions $(16,36)$. The FD in three-dimensional space might be evaluated from several estimates obtained in two-dimensional space, but the procedure was not practical and estimates obtained for two-dimensional projections represented the real plant structure to various degrees. When calculated from a three-dimensional skeletal image, the FD quantifies the structural complexity of a plant root system more accurately $(4,29,30)$. Another obstacle, specific to root system studies, is the opaqueness of the growth medium. Soil opaqueness prevents direct visual observation of the spatial distribution of root systems, the 'hidden half of plants' (39), and critical information about structural complexity is lost when a root system is extracted from the growth medium for observation.

With the advent of nondestructive and noninvasive imaging technologies $(2,38)$, fractal analysis performed from three-dimensional images has recently become possible (30,31). In particular, computed tomography (CT) scanning and magnetic resonance imaging allow the collection of data on objects in three-dimensional space, from which three-dimensional images can be constructed and used as a basis for quantitative analysis at a high level of accuracy. Lontoc-Roy et al. (31) reported one of the first studies of this kind, in which CT scanning was used to follow the development of root systems of maize seedlings in the first days after emergence and to evaluate their complexity from the constructed three-dimensional images.

As a follow-up to the work of Lontoc-Roy et al. (30,31), the objectives of this study were (i) to follow the growth of root systems of control potato plants and of potato plants grown in sand inoculated with a common scab-inducing pathogen, and (ii) to assess the differences between the two groups of potato plants in space occupancy of their underground organs and in structural complexity of their root systems, using CT scan data.

\section{MATERIALS AND METHODS}

Plant, soil preparation, and pathogen inoculation. Potato, the experimental plant, is one of the most important crops in Canada and across the world. It is a typical annual crop, with several orders of roots defined on the basis of their diameter; an illustration of potato first-order roots is given in Figure 1. Shepody, the potato cultivar studied here, was originally bred at the research station of Agriculture and Agri-Food Canada in Fredericton (New Brunswick) in 1969. It has medium-sized spreading roots, and is susceptible to common scab. The seed potatoes (i.e., potatoes from the 2005 harvest) were carefully selected to be exempt of common scab lesions and as much as possible of the same size and shape, and were randomly assigned to the two experimental groups. There were more than three plants in each group, but three plants of each group were thoroughly studied.

The growth medium in this study was fine, homogeneous sand. Before the seed potatoes were potted, sand was sieved to $2 \mathrm{~mm}$ and autoclaved, and kept dry for 1 week prior to filling the pots. Since the dry condition is optimal for isolating roots in sand through CT scan data processing (30), sand was as dry as possible each time CT scan data were collected.

Plants were grown under controlled conditions in a greenhouse (Department of Plant Science, Macdonald Campus, McGill University). They were watered regularly, with Hoagland's nutrient solution $(13,23)$ as modified by Hammer et al. (18) and Wheeler et al. (40), until 3 days before a CT scanning session and immediately after each CT scanning.

Containers were thin plastic pots of a type commonly used in greenhouse work. They were $20 \mathrm{~cm}$ high, and 22 and $18 \mathrm{~cm}$ in diameter at the top and at the bottom, respectively. A common tissue was placed at the bottom to ensure air and water circulation and to prevent sand from going out.

At the beginning of the experiment, a pathogen treatment was applied to the plants of the diseased group (coded A hereafter), whereas the plants of the healthy group were used as controls (C). The common scab-inducing bacterium (S. scabies EF-35; [14]) was inoculated into sand at the time of potting. The $S$. scabies inoculum consisted of $4 \mathrm{ml}$ of Say solution (26), which contained approximately $10^{7}$ spores of the pathogenic strain EF-35. The treated sand filled the space between the seed potato and the periphery of the previously dug cylindrical hole, which was about $10 \mathrm{~cm}$ deep and in the center of the pot. Controls received Say solution alone. Visually (i.e., without the CT scanner), no difference between diseased and healthy plants in emergence from the ground, sprouting, and spreading volume of canopies was perceived.

CT scanning configuration and measurements. X-ray CT scanning technology was originally designed for medical diagnosis to be formulated about internal parts of the human body.

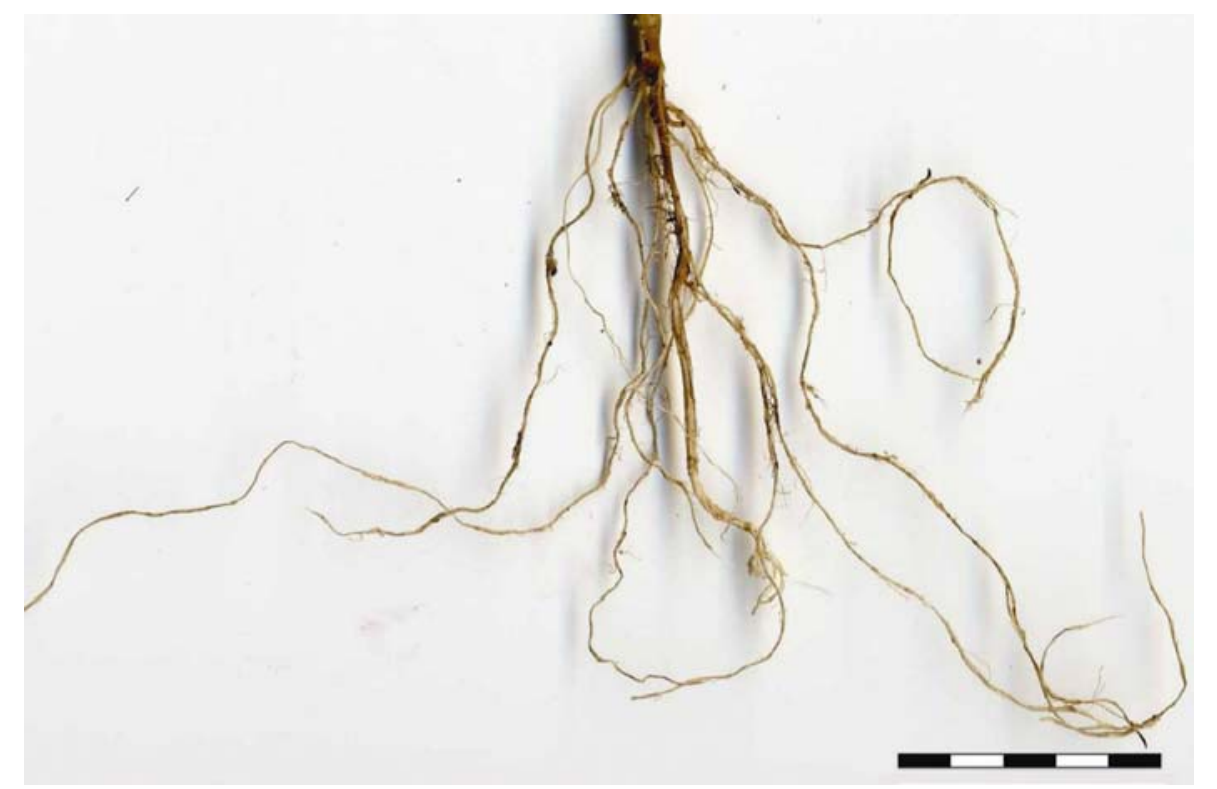

Fig. 1. Digital photograph of the first-order roots of a potato root system after the seed was removed and the hairy roots were washed out. The length of the scale (bottom right) corresponds to $5 \mathrm{~cm}$. 
The Toshiba Xvision high-resolution CT scanner (Toshiba Corporation, Medical Systems Division, Tokyo, Japan) at the CT Scanning Laboratory for agricultural and environmental research on Macdonald Campus of McGill University (Ste-Anne-deBellevue, Québec, Canada) was used to scan the root systems of potted potato plants during their development. The helical scanning mode was chosen for this study, because of its advantages in terms of image reconstruction and advanced interpolation, resulting in enhanced image precision (26). This volumescanning mode also saves time, especially when the objects to be scanned are of relatively large size. To facilitate the later roottracing procedure in CT scan data processing, each potted potato plant was positioned on the side, instead of upright, on the couch of the CT scanner (29).

The CT scanning configuration parameters were defined in accordance with the experimental conditions of the study (e.g., size and matter of the object to be CT scanned, density of the growth medium). In particular, the field of view was $18 \mathrm{~cm}$ in diameter; the zoom factor was set at 1.0 (i.e., no zooming was applied); tube voltage and current were $120 \mathrm{kV}$ and $150 \mathrm{~mA}$, respectively; and the reconstruction interval length was $0.2 \mathrm{~mm}$. In each CT scanning session, a total of 500 cross-sectional images were constructed for each root system and the surrounding growth medium, covering a depth of $10 \mathrm{~cm}$.

A number of functions can be chosen for constructing crosssectional images from CT scan data. With the function that was chosen (i.e., FC70), cross-sectional images were constructed without modifying the raw CT scan data collected. This facilitated data processing in the MATLAB working environment (described below).

Cross-sectional images constructed from CT scan data (example in Fig. 2) are maps of 'CT numbers' (CTN). A CTN is an average relative measure of the density of a pixel in the image, or of the corresponding volume (called 'voxel') with same length and width as the pixel ( $0.35 \mathrm{~mm}$ in this study) and a depth equal to the image construction interval length $(0.2 \mathrm{~mm}$ in this study). A CTN is formally defined as:

$$
\mathrm{CTN}(\mathrm{HU})=\frac{\mu_{\text {object }}-\mu_{\mathrm{water}}}{\mu_{\text {object }}-\mu_{\mathrm{air}}} \times 1000
$$

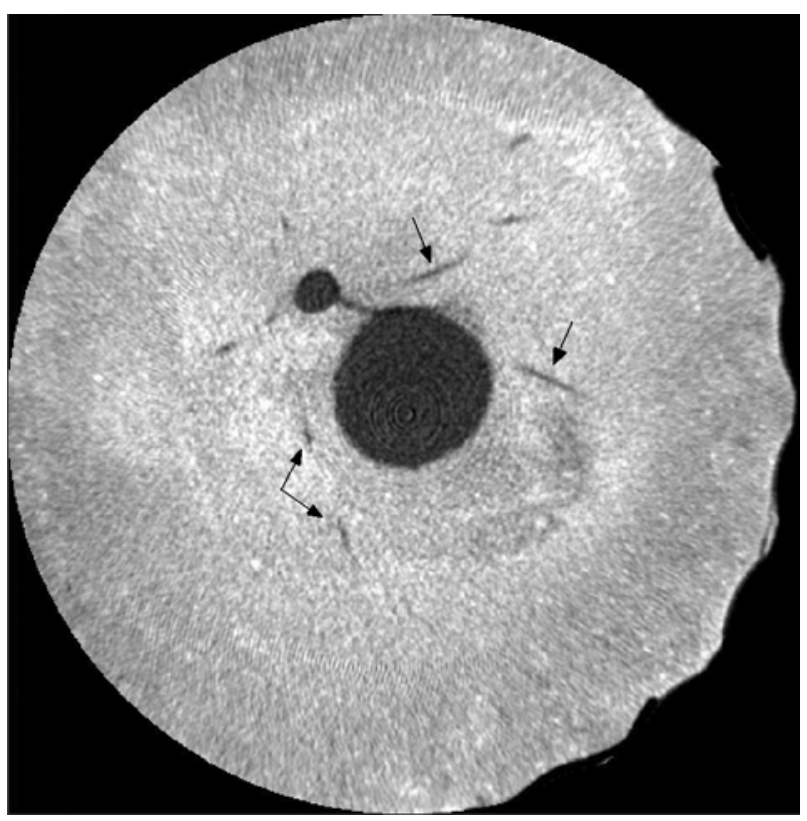

Fig. 2. Cross-sectional computed tomography (CT) image displayed in the root-tracing GUI application program developed in this project. Portions of first-order lateral roots of the potato root system can be seen clearly (as shown with arrows). Physical dimensions of the CT image are $18 \mathrm{~cm} \times 18 \mathrm{~cm}$. where $\mu_{\text {object }}$ is the mean value of the linear attenuation coefficient for the voxel; $\mu_{\text {water }}$ is the linear attenuation coefficient of pure water; and $\mu_{\text {air }}$ is that of a standardized air sample. The unit of CTN is the Hounsfield unit (HU) (26). The CTN scale is linear and centered on 0 , which corresponds to water, while air has the calibrated value of $-1,000 \mathrm{HU}$. Positive and negative CTNs thus represent densities that are greater and less than that of water, respectively.

CT scan data processing and skeletal root image construction. At each scanning time, the raw CTN data, obtained with function FC70 (i.e., 500 slices of $512 \times 512$ pixels and $0.2 \mathrm{~mm}$ thickness), were first displayed on the screen of the CT console and finally processed with an HP workstation xw6000 equipped with a $3.06 \mathrm{GHz}$ Intel Xeon CPU and $2.00 \mathrm{~GB}$ of RAM. In the MATLAB environment (37), the incoming data were converted into an internal data matrix, and a GUI application program designed to visualize cross-sectional images was used to trace the architecture of the first-order root system. First-order roots of potato plants appeared to be more than $1 \mathrm{~mm}$ thick in this study, and could be displayed clearly (Fig. 2). Thus, the center of each first-order root could be located accurately and a list of centers was recorded for each root. Next, the skeleton of each root was constructed using spatial interpolation in three dimensions. Upon completion of this procedure for each individual root, the threedimensional skeletal image of the first-order root system could be assembled and displayed, which was the prerequisite for FD estimation.

Histogram analysis and relative growth rates. Histograms of CT scan data were used as the basis for fitting a model whose parameters provided a measure of space occupancy by the potato underground organs during growth. In a histogram of raw CTN data, two peaks could be distinguished: one of moderate size for potato, starting around $0 \mathrm{HU}$ and stopping before $500 \mathrm{HU}$, and the other for sand, of larger size and located on the right of the potato peak (Fig. 3).

Potato and sand peaks in histograms of raw CTN data were distinctive enough to allow separate curve fitting. Nevertheless, although the growth medium was kept as dry as possible at the time of CT scanning, sand moisture content was not the same for all plants in all weeks, which resulted in varying positions for the sand peak in histograms of raw CTN data. This, in turn, affected the location and shape of the potato peak and any parameter estimates that would follow from the fitting of a curve to it. A

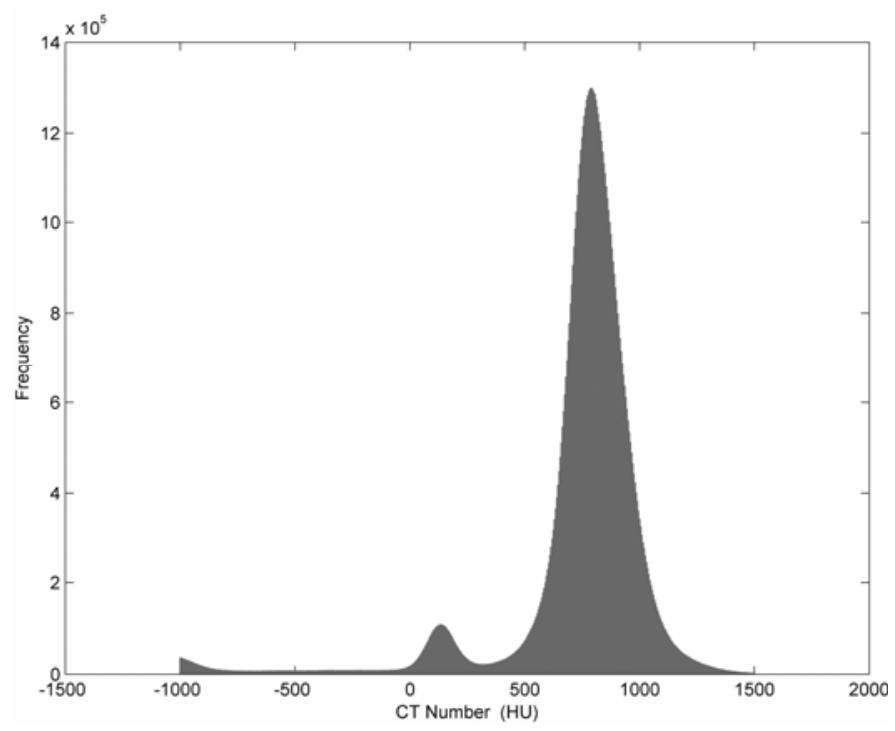

Fig. 3. Histogram of raw computed tomography (CT) numbers for potato plant $\mathrm{C} 3$ in week 4 . The middle secondary peak corresponds to the underground organs, and the main peak on the right, to the growth medium. 
similar effect of soil moisture content (i.e., dry versus watersaturated) was observed on the histograms of raw CTN data when isolating root systems of maize seedlings (30).

Therefore, all sand peaks were standardized to a zero mean and a variance of 1.0, and all data sets were transformed accordingly. More specifically, a bell-shaped curve corresponding to the probability density function of the normal distribution in statistics was first fitted to each sand peak. The equation used for this is

$$
\text { Frequency }=c e^{-\frac{(\mathrm{CTN}-m)^{2}}{v}}
$$

where frequency is the ordinate in the histogram (i.e., the number of CTN data falling in a given class); $m$ and $v$ are, respectively, the parameters of centrality and dispersion of the curve fitted (i.e., classically the population mean and variance in statistical terms); and $c$ is related to the total area under the curve fitted to the peak, which represents a number of voxels and hence, a volume here.

For a given potato plant at a given CT scanning time, parameter estimates $\hat{m}_{\text {sand }}$ and $\hat{v}_{\text {sand }}$ were used to transform the CTN data as follows:

$$
\text { Transformed CTN }=\frac{\mathrm{CTN}-\hat{m}_{\text {sand }}}{\sqrt{\hat{v}_{\text {sand }}}}
$$

This transformation made all potato peaks, from different plants and different weeks, comparable.

A second bell-shaped curve was then fitted, this time to the potato peak in the histogram of transformed CTN data, still using equation (2). The resulting parameter estimates $\hat{v}_{\text {potato }}^{\prime}$ and $\hat{c}_{\text {potato }}^{\prime}$ were used to estimate the volume of space (in number of voxels) occupied by underground organs (roots, seed and stem) at a given stage of development of the potato plant:

$$
\text { Potato volume }=\hat{c}_{\text {potato }}^{\prime} \sqrt{2 \pi \hat{v}_{\text {potato }}^{\prime}}
$$

Note that if $\hat{c}_{\text {potato }}^{\prime}$ was equal to $\left(2 \pi \hat{v}_{\text {potato }}^{\prime}\right)^{-0.5}$, the curve fitted to the potato peak would be exactly the probability density function of a normal distribution; in this hypothetical situation, the area under the curve, and hence potato volume, would be 1.0.

Changes in potato volume over time follow from the growth of roots and the volume loss of the seed. An increased potato volume means that root growth has overcome the loss of volume by the seed potato during the period considered, and vice versa. To take initial differences in volume between seed potatoes into account, relative growth rates (RGR) were used to measure the fluctuations of potato volume (PV) over time (i.e., every 2 weeks):

$$
\mathrm{RGR}_{\text {week } t \text {, week } t+2}=\frac{\mathrm{PV}_{\text {week } t+2}-\mathrm{PV}_{\text {week } t}}{\mathrm{PV}_{\text {week } t}} \text { with } t=4,6
$$

Fractal dimension estimation. A cube counting procedure similar to that of Lontoc-Roy et al. (30) was used for FD estimation; therefore, only the main aspects and specific details of the procedure used in this project are reported below. A new program was written in MATLAB to implement the cube counting procedure. In this program, the binary array representing the three-dimensional skeletal image of a first-order root system is read as input.

The cube counting procedure for FD estimation (32) is based on the counting of cubes that intersect a skeletal image at various scales. More specifically, for a given first-order root system of a potted potato plant CT scanned in a given week, the natural logarithm of the minimum number of intersecting cubes counted for sidelength $s, \log [N(s)]$, is plotted against $\log (1 / s)$, and a straight line with slope $D$ is fitted by simple linear regression with an intercept:

$$
\log [N(s)]=\log K+D \log (1 / s)
$$

where $K$ is a constant. Thus,

$$
N(s) \propto(1 / s)^{D}
$$

By definition, $\mathrm{FD}=D(5)$. If the structure of a root system does follow a fractal pattern, then a strong log-linear relationship between the number of cubes intersecting the three-dimensional skeletal image and the cube sidelength is expected from equation (7). The coefficient of determination $r^{2}$ (where $r$ denotes Pearson's linear correlation coefficient) was used to evaluate the strength of the relationship in equation (6).

The following aspects are specific to the present study. By definition, a cube is a regular hexahedron (i.e., its six faces are squares of same size), but in this study, the basic volumetric unit (i.e., the voxel) is not cubic, since its dimensions are about $0.35 \mathrm{~mm} \times 0.35 \mathrm{~mm} \times 0.2 \mathrm{~mm}$. A ratio of 1.76 (i.e., $0.35 / 0.2$ ) was thus applied to adjust for the unequal sidelengths. This way, the new volumetric unit is a regular hexahedron, as required in the cube counting procedure. In addition, the initial volume containing the three-dimensional skeletal image was repositioned eight times, by shifting it by 0,1 , or 2 voxels in the three directions. The minimum number of intersecting cubes counted for each sidelength was then recorded and retained for further analysis. Other important points are (i) to start with the smallest volume (in the new volumetric unit) containing the three-dimensional skeletal image; (ii) to round to the nearest integer the cube sidelengths successively divided by two only after all divisions were made; and (iii) to fit the regression line of equation (2) by using the four middle points in the log-log plot (30).

Statistical analyses. Two data tables of temporal repeated measures were analyzed statistically. One was made of 12 relative growth rates (between weeks 4 and 6 and between weeks 6 and 8 ), and the other, of 12 FD estimates (in weeks 4 and 6), with treatment as the classification factor (two levels), individual plants in a group as replicates (three plants per treatment), and week defining the repeated measures factor (crossed to treatment). Accordingly, these data tables were analyzed by repeated measures analysis of variance (ANOVA) (10), using the SAS procedure GLM with the option REPEATED (35). Classical $F$ tests were used because the number of repeated measures was only two and the circularity condition was thus satisfied $(25,34)$.

\section{RESULTS}

Root isolation. In this plant CT scan study, the physical dimensions of a voxel were $0.35 \mathrm{~mm} \times 0.35 \mathrm{~mm} \times 0.2 \mathrm{~mm}$, so the first-order roots that had a cross-sectional diameter greater than $0.7 \mathrm{~mm}$ were readily isolated. Direct measurements of root diameter on cross-sectional images supported this. Whereas firstorder roots lateral to the stem could be visualized slice by slice, finer roots branching out from them were rarely seen.

Inspection of potato peaks in histograms. All histograms were plotted using the same scale (Fig. 4). One of the three seed potatoes in the diseased group (A4) clearly was of smaller size than all others. Accordingly, we analyzed potato volume or space occupancy by the underground organs of potato plants during their development, through relative growth rates instead of raw volumes or absolute growth rates. In the same figure, one can see that the left tail of potato peaks, which corresponds to voxels with lighter material, tends to be bigger for healthy plants than for diseased plants (i.e., 617,209 voxels versus 446,518 voxels with a transformed CTN value between -10.0 and -7.0 , on average). A bigger left tail in a potato peak is likely to mean a greater number of roots of second or higher order that were not detected in the first-order root tracing procedure.

Relative growth rates. The repeated measures ANOVA, which covered the period from week 4 to week 8 , involved two relative growth rates for each potato plant. It showed significant treatment main effects at $\alpha=0.05(P=0.0243)$. Very interestingly, mean relative growth rates increased with different sign in the two 
A
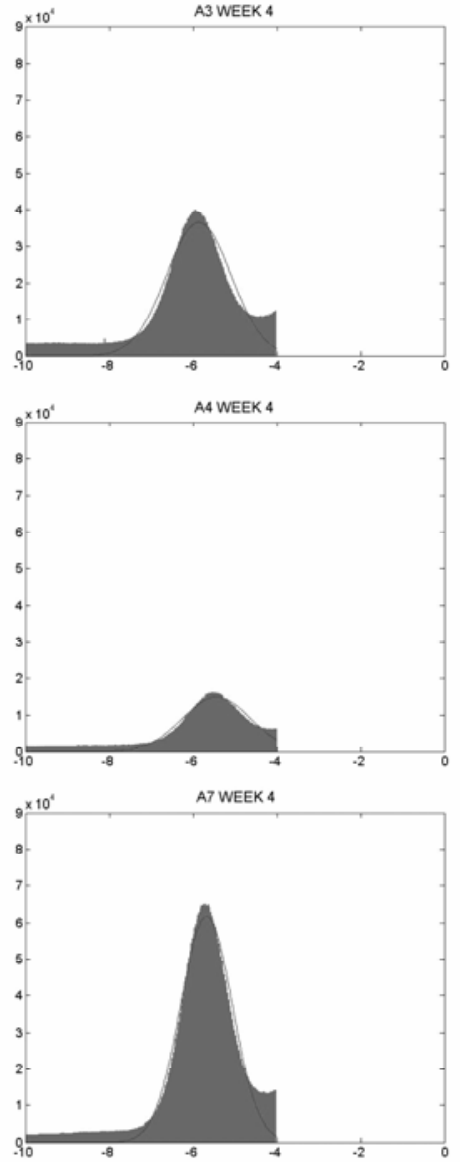

B
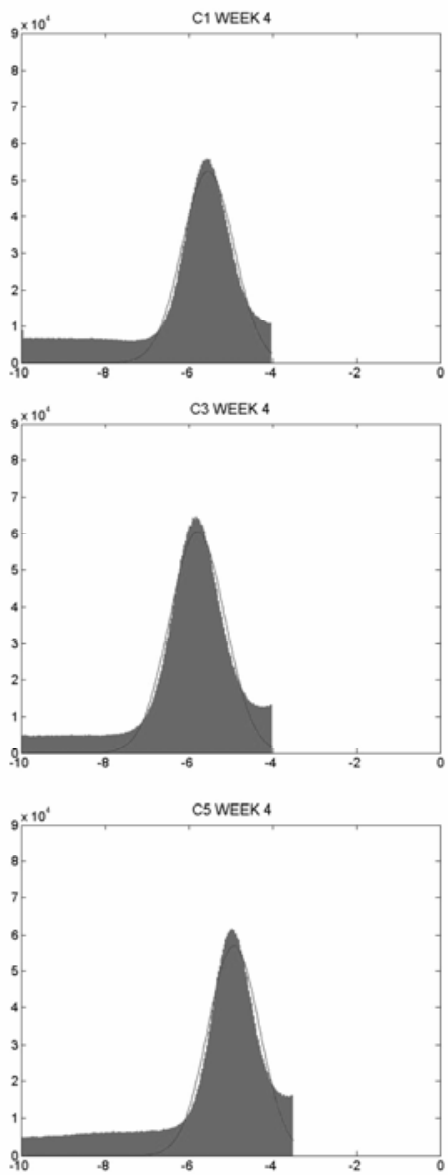
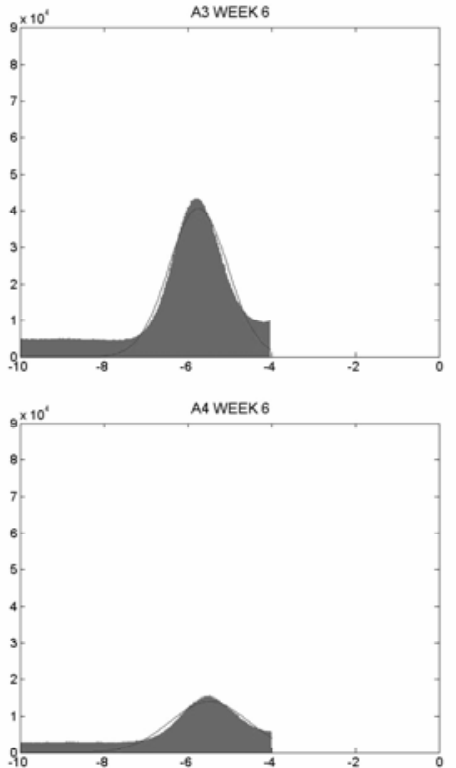

A7 WEEK 6
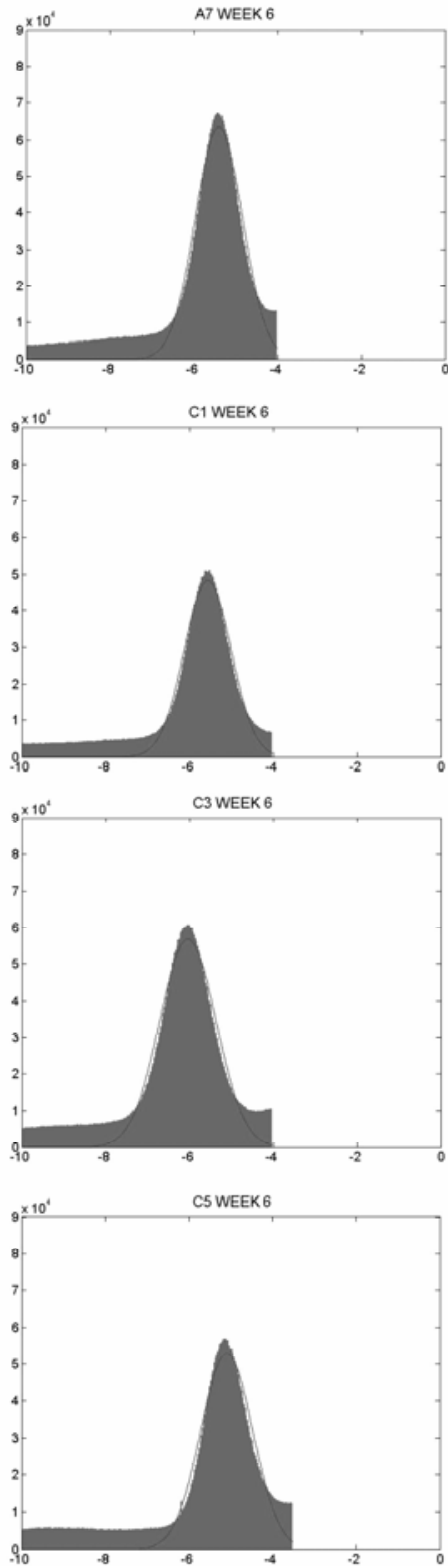
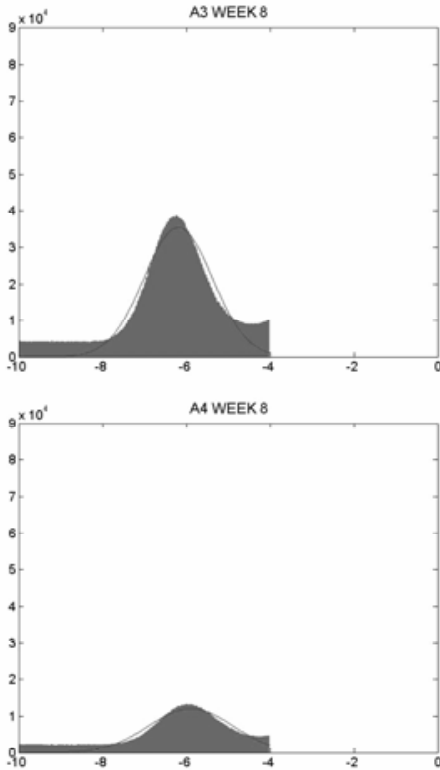

A7 WEEK 8

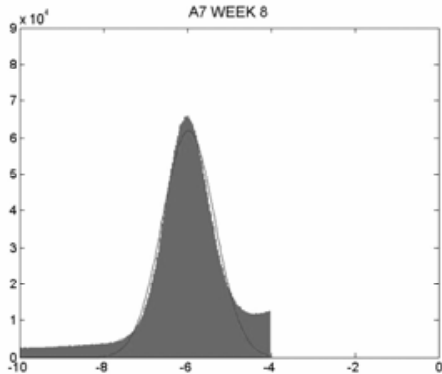

C1 WEEK 8
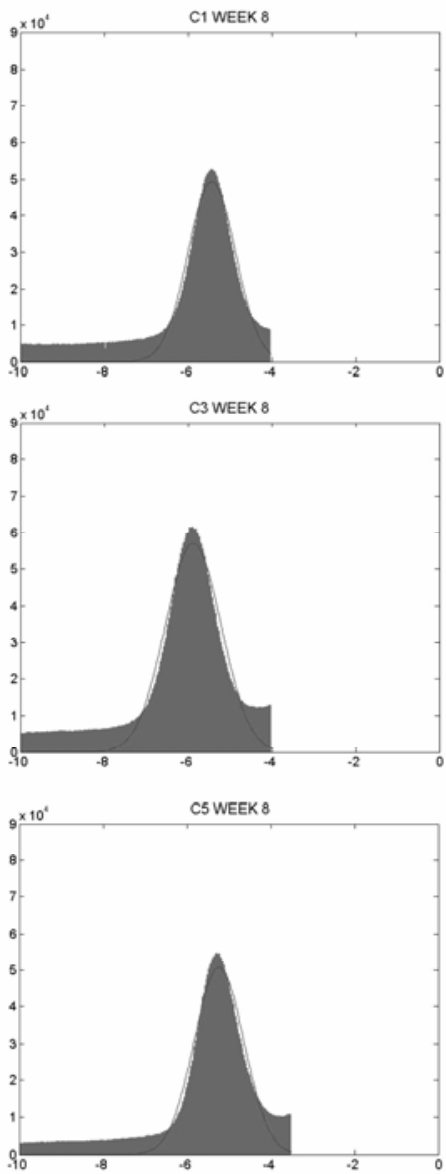

Fig. 4. Potato peaks in the histograms of transformed computed tomography (CT) number data for the potted portion of the $2 \times 3$ individual plants in weeks 4 , 6 , and 8. If displayed, sand peaks would be of greater size than potato peaks and on the right of these. A, Diseased plants; and $\mathbf{B}$, healthy plants. 
TABLE 1. Fractal dimension estimates for the first-order root systems of the six potato plants (A, diseased; $\mathrm{C}$, healthy) in week 4 and week 6 , with the corresponding $r^{2}$ value in parentheses

\begin{tabular}{lcc}
\hline Individual plant & Week 4 & Week 6 \\
\hline A3 & $1.244(0.9980)$ & $1.249(0.9959)$ \\
A4 & $1.275(0.9966)$ & $1.349(0.9939)$ \\
A7 & $1.244(0.9975)$ & $1.307(0.9934)$ \\
C1 & $1.295(0.9941)$ & $1.346(0.9895)$ \\
C3 & $1.310(0.9944)$ & $1.340(0.9914)$ \\
C5 & $1.268(0.9951)$ & $1.348(0.9895)$ \\
\hline
\end{tabular}

groups: from -8.14 to $-0.06 \%$ in healthy plants and from 0.11 to $6.92 \%$ in diseased plants. The about zero mean relative growth rates suggest an almost full energy transfer from the seed, which is shrinking, to the root system, which is growing. The negative mean relative growth rate of $-8.14 \%$ in healthy plants suggests that the new space occupied by growing roots (mainly the firstorder roots) did not compensate for the loss in volume of the seed potato 4 to 6 weeks after planting. As for diseased plants, the positive mean relative growth rate observed at the same period means either a slower decrease in volume of the seed potato or a
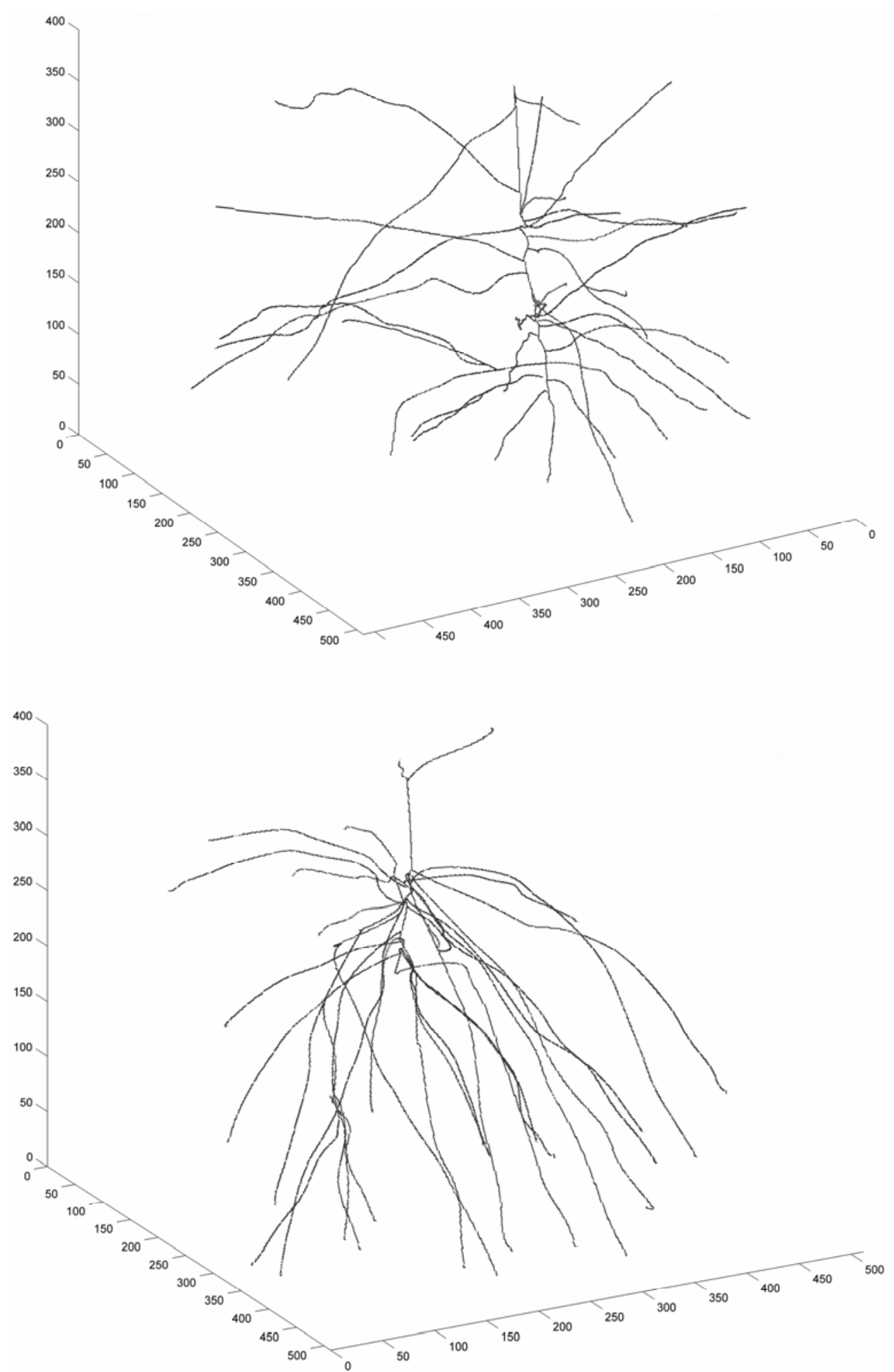

Fig. 5. Three-dimensional skeletal images of first-order root systems of potato plants with lowest (A7 - upper panel) and highest (C3 - lower panel) fractal dimension (FD) estimate in week 4. 
faster growth of roots; either explanation will be confirmed, or not, by the results of fractal analysis of the root systems.

Fractal analysis. The FD estimates obtained for the first-order root systems range from 1.244 to 1.310 in week 4 and from 1.249 to 1.348 in week 8 (Table 1). Though obtained in different manners, these values compare to those obtained in previous root studies $(3,14,26,28,33)$. Unsurprisingly, FD estimates increase from week $4(1.273 \pm 0.027)$ to week $6(1.323 \pm 0.040)$ here. Differences between the two groups of plants appear to exist, especially in week 4. Fractal analysis was not pursued after week 6 because most of the first-order roots had reached the edges of the pot by week 6 , which had affected the architecture of the root system.

Results of the repeated measures ANOVA confirmed and refined the first impression left by the visual inspection of FD estimates (Table 1). Week main effects are significant at $\alpha=0.05$ $(P=0.0170)$ for FD, which reflects the growth and branching of the first-order root systems within the 2 -week interval. Although treatment main effects are not significant overall for FD $(P>$ 0.10 ), the mean values for the two groups in week 4 (A: $1.254 \pm$ 0.018 and $\mathrm{C}: 1.291 \pm 0.021)$ are different at $\alpha=0.10(P=$ 0.0826), which means the disease had a downward effect on the complexity of first-order root systems of potato plants at the early stage of their development (Fig. 5).

\section{DISCUSSION}

In the reported study, the growth of first-order root systems of potted potato plants was followed with a CT scanner of medical type. This technique has the advantages of repeated, noninvasive and nondestructive collection of data on plant material in situ (12). All first-order potato roots that were lateral to the stem and $0.7 \mathrm{~mm}$ thick or more were visualized and traced accurately. Thus, the CT scan data collected allowed the construction of three-dimensional images of developing plant structures, prior to their fractal analysis using the cube counting procedure $(11,30$, 31). This plant CT scan study is the first in which the effects of a pathogen affecting the underground organs were estimated and tested, spatially and temporally, on developing plants. Disease effects on space occupancy were assessed through curve fitting of histograms of appropriately transformed CTN data, and fractal analysis was performed on three-dimensional skeletal images to investigate disease effects on structural complexity. The combination of these two analyses is providing new insight, both graphically and quantitatively, into the symptoms and spread of a plant disease underground, at an unprecedented level of accuracy.

Using relative growth rates calculated from estimated potato volumes, significant differences in space occupancy over time were found between first-order root systems of healthy potato plants and potato plants grown in sand inoculated with a common scab-inducing pathogen. The FD estimates obtained from threedimensional skeletal images showed the increasing complexity of first-order root systems over a 2-week period. Four weeks after planting, a lower complexity was found for diseased potato plants, reflecting a lagged development compared to healthy plants. Each FD estimate contains morphological and topological information about the root system, in terms of root abundance and root branching, so the disease caused a delay in the growth of roots and their branching. In the following 2 weeks, relative growth rates were of different sign between the two groups of plants, suggesting a slower energy transfer in diseased plants between the seed potato and the growing roots during this period. Such delayed growth and slower energy transfer may be related to a surface hardening of the seed potato tissue, due to the disease (19). Previous studies showed that S. scabies induced growth inhibition of seedlings in several crucifers, legumes, and grasses $(17,24,28)$. The weight of soybean and radish grown in the presence of $S$. scabies was significantly reduced compared with noninoculated plants (24). Here, we demonstrated that at the early stage of development, $S$. scabies affects the emergence and growth of roots from the seed potatoes.

The results obtained in this study shed new light into the interactions between potato and a common scab-inducing pathogen. They also provide the basis for future studies with other cultivars and growth media as well as on existing and new control agents or management, including biological control by nonpathogenic streptomycetes (3).

\section{ACKNOWLEDGMENTS}

This project arose from a SEVE Nouvelles Initiatives grant application prepared by the second and fourth authors and awarded to the former in 2005. The first author is also grateful to Centre SEVE (FQRNT Regroupements Stratégiques) for its support in 2006. R. Smith and M. Lapointe provided technical assistance in this project, and special thanks go to Q. Dutilleul for his assistance in the histogram analyses of CT scan data. We thank M. Ngadi, J. Whalen, and two reviewers for constructive comments on various versions of the manuscript.

\section{LITERATURE CITED}

1. Arora, R. K., and Khurana, S. M. P. 2004. Major fungal and bacterial disease of potato and their management. Pages 189-231 in: Fruit and Vegetable Diseases. K. G. Mukerji, ed. Kluwer Academic Publishers, Dordrecht, The Netherlands.

2. Aylmore, L. A. G. 1994. Application of computer assisted tomography to soil-plant-water studies: an overview. Pages 7-15 in: Tomography of SoilWater-Root Processes. S. H. Anderson and J. W. Hopmans, eds. SSSA Special Publication No. 36, Madison, WI.

3. Beauséjour, J., Clermont, N., and Beaulieu, C. 2003. Effect of Streptomyces melanosporofaciens strain EF-76 and chitosan on common scab of potato. Plant Soil 256:463-468.

4. Berntson, G. M. 1994. Root systems and fractals: How reliable are calculations of fractal dimension? Ann. Bot. 73:281-284.

5. Berntson, G. M., Lynch, J. P., and Snapp, S. 1998. Fractal geometry and the description of plant root systems: Current perspectives and future applications. Pages 113-152 in: Fractals in Soil Science. P. Baveye, J. Y. Parlange, and B. A. Stewart, eds. Lewis Publishers, New York.

6. Berntson, G. M., and Woodward, F. I. 1992. The root system architecture and development of Senecio vulgaris in elevated $\mathrm{CO}_{2}$ and drought. Funct. Ecol. 6:324-333.

7. Böhm, W. 1979. Methods of Studying Root Systems. Springer-Verlag, New York.

8. Corbit, J. D., and Garbary, D. J. 1995. Fractal dimension as a quantitative measure of complexity in plant development. Proc. R. Soc. Lon. Ser-B 262:1-6.

9. Costa, C., Dwyer, L. M., Dutilleul, P., Foroutan-pour, K., Liu, A., Hamel, C., and Smith, D. L. 2003. Morphology and fractal dimension of root systems of maize hybrids bearing the leafy trait. Can. J. Bot. 81:706-713.

10. Dutilleul, P. 1998. Incorporating scale into ecological experiments: Data analysis. Pages 387-425 in: Ecological Scale: Theory and Applications. D. L. Peterson and V. T. Parker, eds. Columbia University Press, New York.

11. Dutilleul, P., Han, L., and Smith, D. L. 2008. Plant light interception can be explained via computed tomography scanning. Ann. Bot. 101:19-23.

12. Dutilleul, P., Lontoc-Roy, M., and Prasher, S. O. 2005. Branching out with a CT scanner. Trends Plant Sci. 10:411-412.

13. Epstein, E., and Bloom, A. J. 2005. Mineral Nutrition of Plants: Principles and Perspectives. 2nd ed. Sinauer Associates, Sunderland.

14. Faucher, E., Savard, T., and Beaulieu, C. 1992. Characterization of actinomycetes isolated from common scab lesions on potato tubers. Can. J. Plant Pathol. 14:197-202.

15. Fitter, A. H. 1996. Characteristics and functions of root systems. Pages 120 in: Plant Roots: The Hidden Half, 2nd ed. Y. Waisel, A. Eshel, and U. Kafkafi, eds. Marcel Dekker, New York.

16. Fitter, A. H., and Stickland, T. S. 1992. Architectural analysis of plant root systems. 3. Studies on plants under field conditions. New Phytol. 121:243-248.

17. Goyer, C., Vachon, J., and Beaulieu, C. 1998. Pathogenicity of Streptomyces scabies mutants altered in thaxtomin A production. Phytopathology 88:442-445.

18. Hammer, P. A., Tibbitts, T. W., Langhans, R. W., and MacFarlane, J. C. 1978. Baseline growth studies of 'Grand Rapids' lettuce in controlled environments. J. Am. Soc. Hortic. Sci. 103:649-655.

19. Han, L. 2007. Assessment of common scab effects on the development of potato root systems using computed tomography scanning data. M.Sc. 
thesis, McGill University, Montréal, QC, Canada.

20. Harper, J. L., and Benton, R. A. 1966. The behaviour of seeds in soil: II. The germination of seeds on the surface of a water supplying substrate. J. Ecol. 54:151-166.

21. Harper, J. L., Williams, J. T., and Sagar, G. R. 1965. The behavior of seeds in soil: I. The heterogeneity of soil surfaces and its role in determining the establishment of plants from seed. J. Ecol. 53:273-286.

22. Hill, J., and Lazarovits, G. 2005. A mail survey of growers to estimate potato common scab prevalence and economic loss. Can. J. Plant Pathol. 27:46-52.

23. Hoagland, D. R., and Broyer, T. C. 1936. General nature of the process of salt accumulation by roots with description of experimental methods. Plant Physiol. 11:471-507.

24. Hooker, W. J. 1949. Parasitic action of S. scabies on roots of seedlings. Phytopathology 39:442-462

25. Huynh, H., and Feldt, L. S. 1970. Conditions under which mean square ratios in repeated measurements designs have exact $F$-distributions. J. Am. Stat. Assoc. 65:1582-1589.

26. Kalender, W. A. 2000. Computed Tomography. MCD Verlag, Munich

27. Labruyère, R. E. 1971. Common Scab and Its Control in Seed Potato Crops. Center for Agricultural Publishing and Documentation, Wageningen, the Netherland.

28. Leiner, R. H., Fry, B. A., Carling, D. E., and Loria, R. 1996. Probable involment of thaxtomin A in pathogenicity of Streptomyces scabies on seedlings. Phytopathology 86:709-713.

29. Lontoc-Roy, M. 2005. Three-dimensional visualization in situ and complexity analysis of crop root systems using CT scan data: A primer. M.Sc. thesis, McGill University, Montréal, QC, Canada.

30. Lontoc-Roy, M., Dutilleul, P., Prasher, S. O., Han, L., Brouillet, T., and Smith, D. L. 2006. Advances in the acquisition and analysis of CT scan data to isolate a crop root system from the soil medium and quantify root system complexity in 3-D space. Geoderma 137:231-241.

31. Lontoc-Roy, M., Dutilleul, P., Prasher, S. O., Han, L., and Smith, D. L. 2005. Computed tomography scanning for three-dimensional imaging and complexity analysis of developing root systems. Can. J. Bot. 83:1434-1442.

32. Mandelbrot, B. B. 1983. The Fractal Geometry of Nature. Freeman, New York.

33. Nielson, K. L., Lynch, J. P., and Weiss, H. N. 1997. Fractal geometry of bean root systems: Correlations between spatial and fractal dimension. Am. J. Bot. 84:26-33.

34. Rouanet, H., and Lépine, D. 1970. Comparison between treatments in a repeated-measures design: ANOVA and multivariate methods. Brit. J. Math. Stat. Psy. 23:147-163.

35. SAS Institute, Inc. 2004. SAS for Windows, Release 9.1. SAS Institute, Inc., Cary, NC.

36. Tatsumi, J., Yamauchi, A., and Kono, Y. 1989. Fractal analysis of plant root systems. Ann. Bot. 64:499-503.

37. The MathWorks, Inc. 2006. MATLAB Version R2006. The MathWorks, Inc., Natick, MA.

38. Tollner, E. W., Murphy, C., and Ramseur, E. L. 1994. Techniques and approaches for documenting plant root development with X-ray computed tomography. Pages 115-133 in: Tomography of Soil-Water-Root Processes. S. H. Anderson and J. W. Hopmans, eds. SSSA Special Publication No. 36, Madison, WI.

39. Waisel, Y., and Eshel, A. 2002. Functional diversity of various constituents of a single root system. Pages 157-174 in: Plant Roots: The Hidden Half, 3rd ed. Y. Waisel, A. Eshel, and U. Kafkafi, eds. Marcel Dekker, New York.

40. Wheeler, R. M., Mackowiak, C. L., and Sager, J. C. 1990. Proximate composition of seed and biomass from soybean plants grown at different carbon dioxide $\left(\mathrm{CO}_{2}\right)$ concentrations. TM-103496 Report. NASA, Kennedy Space Center. 